\title{
Creencias sobre Papanicolaou y cáncer cérvicouterino en un grupo de mujeres chilenas
}

\author{
María Teresa Urrutia S. ${ }^{1 a}$ \\ ${ }^{1}$ Departamento Salud de la Mujer, Escuela de Enfermería, Pontificia Universidad Católica de Chile. \\ a Enfermera Matrona, PhD. \\ Proyecto financiado parcialmente por: Sigma Theta Tau International, Beta Tau Chapter.
}

\section{RESUMEN}

Antecedentes: Son variadas las descripciones en relación a las creencias y como estas se relacionan con las conductas preventivas en cáncer cérvicouterino. Objetivo: Describir las creencias que tienen un grupo de mujeres chilenas pertenecientes al sistema público de atención acerca del Papanicolaou y cáncer cérvicouterino. Método: Estudio analítico de corte transversal realizado en 333 mujeres chilenas. Se estudiaron las creencias sobre el Papanicolaou y el cáncer cérvicouterino con un instrumento desarrollado y validado en población chilena (CPC-28). Resultados: El 96\% de las mujeres refiere tener un Papanicolaou en los últimos 3 años. El antecedente familiar es reportado por el 49,8\% como causa de cáncer cérvicouterino. La barrera principal para adherir al Papanicolaou es la falta de conocimiento en cuanto a la edad requerida. La principal señal de acción que impulsa a que la mujer adhiera al tamizaje es la indicación entregada por el doctor. El beneficio mas importante es el cuidado de la salud. El 14,1\% señala la presencia de relaciones sexuales como necesaria para adherir al tamizaje. El 17,4\% de las mujeres se percibe fuera de riesgo de desarrollar un cáncer cérvicouterino. Las 6 dimensiones estudiadas se correlacionan entre si. Conclusión: Las creencias deben ser consideradas al momento de intervenir una población, empezando por valorarlas y comprenderlas para posteriormente poder modificarlas.

\section{PALABRAS CLAVE: Cáncer cérvicouterino, Papanicolaou, creencias, modelo de creencias en salud}

\section{SUMMARY}

Background: Many reasons has been described about relation between beliefs and cervical cancer behaviors. Objective: To describe the beliefs about cervical cancer and Pap test in a group of chilean women. Method: Cross sectional and analytic study was done with 333 chilean women. Beliefs about cervical cancer and Pap test was studied with the CPC-28 questionnaire, developed and validated in Chilean population. Results: $96 \%$ have had a Pap test in the last 3 years. Family history about cervical cancer was reported by $49.8 \%$ as a cause of cervical cancer. The principal barrier to Pap test was lack of knowledge about the age. The principal cue to action was the doctor recommendation. The benefit was the care of health. The sexual intercourse was reported by $14.1 \%$ as necessary to take a Pap test. The risk to develop cervical cancer is reported by $17.4 \%$. The six dimensions studied were correlated between them. Conclusion: The beliefs must be considered to education programs, firstly understanding and then modifying them.

KEY WORDS: Cervical cancer, Pap test, beliefs, health belief model 


\section{INTRODUCCIÓN}

El cáncer cérvicouterino (CC) es uno de los canceres con la más alta mortalidad en el mundo (1). Cada año 33.000 mujeres mueren en Latinoamérica y el Caribe (2) por esta causa. En Chile el CC ha sido la causa principal de años de vida potencialmente perdidos (3).

Si bien la vacunación contra el virus Papiloma Humano (VPH) es un recurso disponible, no es accesible a toda la población y por otro lado no reemplaza las estrategias de tamizaje $(2,4,5)$. Por lo anterior, es necesario conocer aquellos aspectos que impiden que las mujeres adhieran al tamizaje, y entre esos aspectos se encuentran las creencias que la población tiene acerca del Papanicolaou (Pap) y del CC.

A nivel internacional, son variadas las descripciones en relación a las creencias y como estas se relacionan con las conductas preventivas en CC $(6,7,8)$. En Chile la aproximación ha sido realizada como falta de conocimientos (9), formas de prevención adoptadas por la población (10), y barreras para adherir al Pap $(11,12)$, sin embargo, ningún estudio ha abordado la problemática desde la perspectiva de un modelo teórico, abarcando los diferentes componente que las creencias tienen y como están relacionadas entre sí.

El modelo de creencias en salud señala que la forma de conocer y comportarse está influenciada por las creencias y emociones de las personas, las que a su vez determinan las decisiones en salud $(13,14)$. Este modelo fue desarrollado para explicar el porqué las personas no adhieren a conductas preventivas relacionadas con la detección temprana de una enfermedad $(13,15,16)$.

El objetivo de este estudio es describir las creencias que tienen un grupo de mujeres chilenas pertenecientes al sistema público de atención acerca del Pap y CC.

\section{PACIENTES Y MÉTODO}

Estudio analítico de corte transversal realizado en un grupo de mujeres chilenas. La muestra de tipo intencionada está constituida por 333 mujeres que se atendieron en el servicio de ginecología del Complejo Asistencial Dr. Sotero del Rio (CADSR) durante el periodo comprendido entre el 20 de abril y 20 de mayo 2009. Los criterios de inclusión fueron ser usuaria del CADSR y edad entre 18 y 64 años. Se excluyeron aquellas mujeres histerectomizadas y con antecedentes personales de patología cervical.
Las variables incluidas en este estudio fueron variables socio-demográficas, antecedentes sobre CC y creencias sobre el Pap y el CC. La variable creencia fue medida con un instrumento desarrollado y validado en población chilena (CPC-28) (17) basado en el modelo de creencias en salud descrito por Rosenstock $(13,16,18)$. El CPC-28 presenta 6 dimensiones: barreras ( 9 ítems), beneficios (3 ítems), severidad (4 ítems), susceptibilidad (3 ítems), señales de acción (6 ítems) y requisitos (3 ítems). El alpha de Cronbach en la presente muestra fue 0,74 .

Esta investigación contó con la aprobación de los comités de ética del Servicio de Salud Metropolitano Sur-Oriente en Chile y la Universidad de Miami en EEUU. El análisis de los datos se realizó con el programa SPSS, 18.0. Se realizaron medidas de tendencia central, dispersión, análisis de frecuencia, y correlación entre factores mediante análisis factorial. Cabe señalar que las correlaciones factoriales son consideradas bajas si los valores son menores de 0,20 y altas cuando son mayores a 0,50 . Correlaciones factoriales superiores a 0,50 indican la necesidad de fusionar ambos factores (19).

\section{RESULTADOS}

El promedio de edad de las mujeres fue de 38 $\pm 10,8$ años, con un rango entre 18 y 64 años de edad. El promedio de años de escolaridad fue de $11 \pm 2,4$ años. El 67,6\% de la muestra declara tener pareja, y el $86 \%$ tener hijos.

El $17 \%$ de las mujeres señala tener o haber tenido un familiar o conocido con CC. El $96 \%$ de las mujeres entre 24 y 64 años de edad refiere tener un Pap en los últimos 3 años, presentando el 57\% de ellas el Pap el último año. Al consultar sobre las causas que provocan CC, el principal motivo identificado fue el antecedente familiar, con el 49,8\% de las mujeres en estudio (Tabla I). El 97\% de las mujeres señala que si la vacuna contra el VPH estuviera disponible, ellas se la colocarían.

En relación a las barreras para adherir al tamizaje, el principal fue la falta de conocimiento en cuanto a la edad requerida, seguido por la falta de conocimiento en cuanto a la frecuencia que debe tomarse el Pap y en tercer lugar el largo tiempo de espera para ser atendida (Tabla II).

La principal señal de acción que impulsa a que la mujer adhiera al tamizaje es la indicación entregada por el doctor. En relación a los beneficios percibidos, el más importante es el cuidado de la salud (Tabla III). Al analizar las creencias en cuanto a los 
Tabla I

\section{CAUSAS DE CÁNCER CÉRVICOUTERINO REPORTADAS POR LAS MUJERES}

\begin{tabular}{lcc}
\hline Causa & $\begin{array}{c}\text { Respuestas } \\
\text { entregadas }\end{array}$ & $\begin{array}{c}\text { \% de la } \\
\text { muestra }\end{array}$ \\
\hline Antecedentes familiares & 166 & 49,8 \\
Un virus & 133 & 39,9 \\
Relaciones sexuales & 92 & 27,6 \\
La mala suerte & 38 & 11,4 \\
El envejecimiento & 16 & 4,8 \\
Total & 445 & - \\
\hline
\end{tabular}

(*) El número de respuestas supera el total de la muestra en estudio dado que una mujer puede dar más de una respuesta.

requisitos para adherir al Pap, se observa que el $14,1 \%$ de la muestra reporta que la presencia de relaciones sexuales es necesaria para adherir al tamizaje (Tabla IV),

Al analizar la severidad de la enfermedad percibida por las mujeres, el $96 \%$ de las mujeres señala que el CC es un problema serio de salud. En relación a la susceptibilidad (riesgo de desarrollar un cáncer), el $17,4 \%$ de ellas se percibe fuera de riesgo de desarrollar un CC (Tabla IV).

Al hacer el análisis de los 6 factores del instrumento mediante análisis factorial (Tabla V), se observa la relación entre las diferentes dimensiones estudiadas. Cada uno de los factores tiene a lo menos una correlación con valores entre 0,20 y 0,50.

Al observar las correlaciones, todas ellas, salvo una, son positivas, lo que indica que las dimensiones en estudio se mueven en una misma dirección. Es así como la relación entre las 6 dimensiones se interpreta de la siguiente forma:

- Mientras más barreras para adherir al tamizaje tenga la mujer, la creencia que debe cumplir determinados requisitos también es alta, y viceversa.

- Las señales de acción son eficientes cuando existe mayor susceptibilidad y mayores beneficios percibidos por la mujer, y viceversa

- A mayor severidad percibida por la mujer es mayor la susceptibilidad y los beneficios que la mujer percibe al adherir al tamizaje, y viceversa.

- A mayor requisitos percibidos para adherir al tamizaje los beneficios disminuyen, y viceversa

- A mayor susceptibilidad percibida mayores son los beneficios, y viceversa.

Cabe señalar que al ser análisis de correlaciones, la dirección causal no es posible de ser inferida.

Tabla II

BARRERAS PARA ADHERIR AL TAMIZAJE REPORTADAS POR LAS MUJERES

\begin{tabular}{lcccc}
\hline Ítems & $\begin{array}{c}\text { Muy de } \\
\text { acuerdo } \\
(\%)\end{array}$ & $\begin{array}{c}\text { De } \\
\text { acuerdo } \\
(\%)\end{array}$ & $\begin{array}{c}\text { En } \\
\text { desacuerdo } \\
(\%)\end{array}$ & $\begin{array}{c}\text { Muy en } \\
\text { desacuerdo } \\
(\%)\end{array}$ \\
\hline Yo no sé a qué edad es necesario tomarse el Pap & 13,3 & 20,0 & 34,5 & 32,1 \\
$\begin{array}{l}\text { Yo no sé cada cuanto tiempo necesito ir a tomar- } \\
\text { me el Pap }\end{array}$ & 10,8 & 15,6 & 38,7 & 34,8 \\
$\begin{array}{l}\text { No me tomo el Pap porque cuando voy necesito } \\
\text { esperar largo tiempo para ser atendida }\end{array}$ & 13,2 & 12,9 & 39,3 & 34,5 \\
$\begin{array}{l}\text { No me tomo el Pap porque cuesta mucho sacar } \\
\text { una hora de atención }\end{array}$ & 9,4 & 9,7 & 41,2 & 39,7 \\
$\begin{array}{l}\text { No me tomo el Pap porque el consultorio atiende } \\
\text { en horarios en los que no puedo ir }\end{array}$ & 8,4 & 9 & 35,2 & 47,3 \\
$\begin{array}{l}\text { No me tomo el Pap porque me da miedo saber } \\
\text { que tengo cáncer }\end{array}$ & 7,2 & 9,3 & 32,7 & 50,8 \\
$\begin{array}{l}\text { No me tomo el Pap porque me da vergüenza que } \\
\text { me examinen los genitales }\end{array}$ & 5,1 & 6,9 & 31,2 & 56,8 \\
$\begin{array}{l}\text { No me tomo el Pap porque en el consultorio me } \\
\text { tratan mal }\end{array}$ & 4,8 & 6,0 & 40,8 & 48,3 \\
No tengo tiempo para tomarme el Pap & 5,2 & 5,5 & 37,8 & 51,5 \\
\hline
\end{tabular}




\section{Tabla III}

\section{SEÑALES DE ACCIÓN (MOTIVACIÓN) PARA ADHERIR AL TAMIZAJE REPORTADAS POR LAS MUJERES}

\begin{tabular}{|c|c|c|c|c|c|}
\hline Dimensión & Ítems & $\begin{array}{l}\text { Muy de } \\
\text { acuerdo }\end{array}$ & $\begin{array}{l}\text { De } \\
\text { acuerdo }\end{array}$ & $\begin{array}{c}\text { En } \\
\text { desacuerdo }\end{array}$ & $\begin{array}{c}\text { Muy en } \\
\text { desacuerdo }\end{array}$ \\
\hline \multirow{6}{*}{$\begin{array}{l}\text { Señales de acción } \\
\text { para adherir al tamizaje }\end{array}$} & Porque un doctor me lo pide & $31 \%$ & $36,7 \%$ & $20,5 \%$ & $11,7 \%$ \\
\hline & $\begin{array}{l}\text { Porque una enfermera o matrona } \\
\text { me lo pide }\end{array}$ & $32,4 \%$ & $32,4 \%$ & $22,8 \%$ & $12,3 \%$ \\
\hline & $\begin{array}{l}\text { Porque escuche o lei en el diario } \\
\text { o en algún programa de televisión } \\
\text { o radio }\end{array}$ & $28,8 \%$ & $32,1 \%$ & $24,3 \%$ & $14,7 \%$ \\
\hline & $\begin{array}{l}\text { Porque mi madre me habla sobre } \\
\text { eso }\end{array}$ & $28,5 \%$ & $30,3 \%$ & $26,1 \%$ & $15 \%$ \\
\hline & $\begin{array}{l}\text { Porque miembros de mi familia } \\
\text { me dijeron que lo tomara }\end{array}$ & $23,7 \%$ & $27,6 \%$ & $30,3 \%$ & $18,3 \%$ \\
\hline & $\begin{array}{l}\text { Porque mi amiga o vecina me } \\
\text { habla sobre eso }\end{array}$ & $21,3 \%$ & $27,9 \%$ & $33,9 \%$ & $16,8 \%$ \\
\hline \multirow[t]{3}{*}{$\begin{array}{l}\text { Beneficios de adherir } \\
\text { al tamizaje }\end{array}$} & $\begin{array}{l}\text { Tomarme el Pap me hace sentir } \\
\text { bien porque significa que yo } \\
\text { cuido de mi salud }\end{array}$ & $83,2 \%$ & $15,9 \%$ & $0,6 \%$ & $0,3 \%$ \\
\hline & $\begin{array}{l}\text { Una razón para tomarme el Pap } \\
\text { es cuidar de mi salud }\end{array}$ & $78,4 \%$ & $20,4 \%$ & $0,3 \%$ & $0,9 \%$ \\
\hline & El Pap puede salvar mi vida & $78,9 \%$ & $18,1 \%$ & $0,9 \%$ & $2,1 \%$ \\
\hline \multirow[t]{3}{*}{$\begin{array}{l}\text { Requisitos para adherir } \\
\text { al tamizaje }\end{array}$} & $\begin{array}{l}\text { Si no he tenido hijos, no necesito } \\
\text { tomarme el Pap }\end{array}$ & $5,1 \%$ & $7,5 \%$ & $43,7 \%$ & $43,7 \%$ \\
\hline & $\begin{array}{l}\text { Si no estoy teniendo relaciones } \\
\text { sexuales, no necesito tomarme } \\
\text { el Pap }\end{array}$ & $6 \%$ & $8,1 \%$ & $46,8 \%$ & $39 \%$ \\
\hline & $\begin{array}{l}\text { Si no tengo síntomas o molestias, } \\
\text { no necesito tomarme el Pap }\end{array}$ & $5,1 \%$ & $5,7 \%$ & $43,5 \%$ & $45,6 \%$ \\
\hline
\end{tabular}

\section{DISCUSIÓN}

La fortaleza del presente estudio es ser el primero que reporta las creencias de un grupo de mujeres chilenas, basado en el modelo de creencias en salud, en relación al CC y Pap. Estos resultados permitirán contribuir a la valoración de aspectos centrales al momento de estudiar las causas de fracasos del programa de prevención de CC en Chile.

El análisis de la adherencia al tamizaje, en cuanto al porcentaje de mujeres con Pap al día, debe ser visto con precaución, fundamentalmente por las características de la muestra en estudio. Las mujeres de este grupo son usuarias en control activo en un servicio de ginecología, lo que permite por un lado estar en contacto con profesionales del área, sensibilizándose al tema en estudio, y por otro la posibilidad que haya una indicación concreta del examen. Ambas razones pudieran estar explicando la alta tasa reportada, la que se sin duda no corresponde a la realidad nacional (20). Cabe señalar además que estudios de auto reporte señalan que el rango de error es entre un 20 a $25 \%$, siendo mayor el porcentaje a medida que aumenta el rango de tiempo a recordar $(21,22)$. Por lo anterior se puede inferir que el porcentaje de mujeres con Pap al día podría disminuir llegando a $71 \%$, cifra más cercana a la realidad nacional, sin embargo, no igual.

Otro aspecto a considerar en el análisis de los resultados, es el $17 \%$ de mujeres que reporta tener o haber tenido un familiar o amiga con CC. Este aspecto ha sido descrito como un factor que incrementa la percepción de susceptibilidad o riesgo a desarrollar un CC $(7,8)$. Por otro lado, Boyer y cols (23), en un estudio realizado en mujeres latinas señalan que el contacto con mujeres con $\mathrm{CC}$ hace que la adherencia al tamizaje aumente.

Es muy interesante el hallazgo que casi la mitad 
Tabla IV

SEVERIDAD DEL CÁNCER CÉRVICOUTERINO Y SUSCEPTIBILIDAD PERCIBIDA PARA DESARROLLARLO REPORTADA POR LAS MUJERES

\begin{tabular}{|c|c|c|c|c|c|}
\hline Dimensión & Ítems & $\begin{array}{l}\text { Muy de } \\
\text { acuerdo }\end{array}$ & $\begin{array}{l}\text { De } \\
\text { acuerdo }\end{array}$ & $\begin{array}{c}\text { En } \\
\text { desacuerdo }\end{array}$ & $\begin{array}{c}\text { Muy en } \\
\text { desacuerdo }\end{array}$ \\
\hline \multirow[t]{4}{*}{ Severidad } & $\begin{array}{l}\text { El cáncer cérvicouterino es un } \\
\text { problema de salud serio }\end{array}$ & $70,3 \%$ & $25,8 \%$ & $0,9 \%$ & $3 \%$ \\
\hline & $\begin{array}{l}\text { El cáncer cérvicouterino puede } \\
\text { llevar a una mujer a tener que reali- } \\
\text { zarse un tratamiento con quimiote- } \\
\text { rapia o radioterapia }\end{array}$ & $60,2 \%$ & $34,3 \%$ & $3,3 \%$ & $2,1 \%$ \\
\hline & $\begin{array}{l}\text { El cáncer cérvicouterino puede } \\
\text { llevar a una mujer a tener que so- } \\
\text { meterse a una histerectomía }\end{array}$ & $58,9 \%$ & $30,6 \%$ & $6,6 \%$ & $3,9 \%$ \\
\hline & $\begin{array}{l}\text { El cáncer cérvicouterino puede } \\
\text { causar la muerte }\end{array}$ & $61 \%$ & $26,4 \%$ & $6 \%$ & $6,6 \%$ \\
\hline \multirow[t]{3}{*}{ Susceptibilidad } & $\begin{array}{l}\text { Yo tengo riesgo de desarrollar un } \\
\text { cáncer cérvicouterino }\end{array}$ & $30,5 \%$ & $35,1 \%$ & $17,1 \%$ & $17,4 \%$ \\
\hline & $\begin{array}{l}\text { Si yo tengo cáncer cérvicouterino } \\
\text { me puedo morir }\end{array}$ & $45,6 \%$ & $34,5 \%$ & $12,9 \%$ & $6,9 \%$ \\
\hline & $\begin{array}{l}\text { El cáncer cérvicouterino es uno de } \\
\text { los cánceres más comunes entre } \\
\text { las mujeres de mi edad }\end{array}$ & $40,5 \%$ & $39,6 \%$ & $11,8 \%$ & $8,2 \%$ \\
\hline
\end{tabular}

Tabla V

MATRIZ DE CORRELACIÓN FACTORIAL, INSTRUMENTO CPC28 (*)

\begin{tabular}{lcccccc}
\hline Factor & Barrera & $\begin{array}{c}\text { Señal de } \\
\text { acción }\end{array}$ & Severidad & Requisitos & Susceptibilidad & Beneficios \\
\hline Barreras & 1 & - & - & 0,339 & - & - \\
Señal de acción & - & 1 & - & - & 0,210 & 0,241 \\
Severidad & - & - & 1 & - & 0,446 & 0,354 \\
Requisitos & 0,339 & - & - & 1 & - & $-0,314$ \\
Susceptibilidad & - & 0,210 & 0,446 & - & 1 & 0,288 \\
Beneficios & - & 0,241 & 0,354 & $-0,314$ & 0,288 & 1 \\
\hline
\end{tabular}

$\left(^{\star}\right)$ Solo se muestran las correlaciones factoriales mayores de 0,20 .

de la muestra en estudio considera la historia familiar de CC como una causa del mismo. Este hallazgo puede responder al conocimiento tradicional de otros tipos de cáncer y su asociación a la genética, sin embargo, es sabido que el antecedente familiar no es un factor de riesgo de CC (24), por lo que este hallazgo debiera ser considerado al momento de educar a la población para corregir el error. La creencia errada en cuanto a este factor de riesgo hará que, en el caso de las mujeres que no tienen antecedentes, no se sientan susceptibles a desa- rrollar esta enfermedad y por ende no sientan la necesidad de adherir al tamizaje. Si a lo anterior se agrega que solo un $40 \%$ de las mujeres asocia el CC a un virus y menos del $30 \%$ de ellas a la actividad sexual, la importancia de educar sobre factores de riesgo para desarrollar un CC cobra mayor relevancia. Es sabido que casi todos los casos de CC están asociados al VPH, el cual es transmitido por vía sexual (24-26).

En relación a las barreras que las mujeres señalan, llama la atención que en general no más del 
$33 \%$ de las mujeres declara estar de acuerdo o totalmente de acuerdo con alguna de las barreras, lo que podría estar explicado por las características de la muestra, ya que son personas adherentes a controles médicos ginecológicos, y en su mayoría con el Pap al día. Sin embargo, dentro de las barreras reportadas, las más importantes dicen relación a la falta de información respecto de los requisitos y la atención del centro de salud.

En relación a la primera barrera, en un estudio realizado con población latina, el porcentaje de mujeres que desconocía la periodicidad con que debe hacerse el examen llego al $21 \%$ (27), cifra levemente inferior al del presente estudio. En relación a la edad, en un estudio realizado en Tailandia (28), se reportó que el $82,5 \%$ de la población conocía el rango de edad, cifra casi 20 puntos porcentuales mayor al porcentaje obtenido en esta muestra. La claridad en cuanto a estos dos requerimientos es otro punto importante a considerar en la educación de las usuarias. Si ellas no cuentan con esta información mínima, difícilmente podremos esperar que adhieran al programa de prevención.

Respecto de las barreras relacionadas al centro de salud: los horarios, el largo tiempo de espera y la dificultad en obtener una hora de atención son las barreras descritas que podrían agruparse en problemas de acceso a tamizaje, problemas que han sido descritos también por otros autores $(23,29)$. Esta información es importante para aquellos profesionales de la administración de los centros primarios de atención, para permitir una mayor flexibilidad al momento de considerar el acceso de las mujeres al tamizaje. Si bien se han tomado medidas, siguen siendo estos aspectos percibidos como barreras por parte de las mujeres usuarias del sistema.

El miedo y la vergüenza han sido descritos previamente como barreras en otros grupos étnicos $(7,8,30)$, demostrando lo transversal que son culturalmente las dos emociones. El miedo también fue reportado en dos estudios chilenos previos $(11,12)$.

En relación a las señales de acción, destaca la importancia que alcanza la indicación realizada por los profesionales de la salud. La indicación médica tiene peso al momento de llevar a cabo una conducta preventiva, siendo vista como un factor reforzador de la conducta (31) o incluso una inspiración para la misma (32). La importancia de dar la orden escrita y verbal para el examen de Pap debiera ser asumido no tan solo por los profesionales del área, sino por todos aquellos profesionales de la salud, independiente del lugar o motivo de consulta de la mujer. Cualquier contacto en salud debiera ser aprovechado para invitar a la mujer a adherir al tamizaje.

La percepción de severidad de la enfermedad es bastante alta en este grupo de estudio. Más del $80 \%$ de las mujeres reconoce las consecuencias que tiene el CC en cuanto a alternativas terapéuticas (como la histerectomía, quimioterapia, radioterapia), seriedad de la enfermedad e incluso la muerte como consecuencia. Al respecto es importante señalar que la muerte puede por una lado favorecer a que la mujer adhiera al tamizaje, pero por otro lado también puede alejarla de la práctica preventiva ya que es tomada como una "sentencia" $(33,34)$ y por lo tanto considerarlo fatal sin posibilidad de hacer algo (8). El fatalismo (35) es un valor cultural propio de los latinos, que nuestro país comparte, y que debe ser tratado con mucha cautela al momento de educar a la población. La fatalidad de una enfermedad si es tomado en su expresión máxima, evitara que la mujer lleve a cabo una conducta preventiva, asegurando que "frente a dicha sentencia de muerte no hay nada que hacer".

Finalmente al analizar las correlaciones entre las 6 dimensiones del instrumento utilizado, queda en evidencia aquellos aspectos que deben ser considerados al momento de establecer un programa educativo, basado en creencias. En primer lugar debiera clarificarse los requisitos, para que las mujeres puedan adherir al tamizaje de manera informada, evitando confusiones en cuanto a edad, frecuencia y por sobre todo sobre la necesidad de estar sexualmente activa, tener hijos o presentar síntomas. Mientras menos requisitos se perciben como necesarios, mayores serán los beneficios de adherir al tamizaje.

El trabajo educativo sobre la susceptibilidad a desarrollar un CC es crucial. Para ello es necesario que se converse con la mujer sobre los factores de riesgo, y por ende desmitificar la idea de que es necesario tener antecedentes familiares de CC para poder desarrollarlo. El aumento en la percepción de riesgo, permitirá que los beneficios sean percibidos con mayor fuerza, y que las señales de acción, como la indicación médica de hacerse el examen, sean más efectivas.

Respecto de la severidad de la enfermedad, esta creencia se mueve en la misma dirección que la susceptibilidad, por lo tanto la educación sobre los factores de riesgos y consecuencias de la enfermedad debieran contribuir a la adherencia del tamizaje, ya que ambos aumentan la percepción de beneficios. En este punto cobra importancia la adecuación en la intensidad, ya que un exceso de severidad percibida, conecta a la mujer con la fatalidad, y por ende con la no adherencia.

La Real Academia de la Lengua Española define creencia como el "firme asentimiento y conformidad con algo" (36), por lo tanto si un "firme asentimiento" 
sobre algún tema en salud hace que el usuario actúe alineado a dicha creencia, y por lo tanto desechando aquellos aspectos que no coinciden con ella, cualquier estrategia educativa que no tome en cuenta las creencias no podrá ser llevada a cabo con éxito.

\section{CONCLUSIÓN}

Para el éxito de un programa preventivo en salud, las creencias deben ser consideradas al momento de intervenir una población, empezando por valorarlas y comprenderlas para posteriormente poder modificarlas.

\section{REFERENCIAS}

1. Organización Panamericana de la Salud. Prevención y detección temprana pueden evitar una escalada de muertes por cáncer en las Américas. 2007. Disponible en: http://www.paho.org/Spanish/DD/PIN/ps070204. htm. Consultado el 1 de septiembre de 2008.

2. Organización Panamericana de la Salud. Expertos en la Américas piden mejoras para evitar muertes por cáncer de cuello uterino. 2008. Disponible en: http:// www.paho.org/Spanish/DD/PIN/ps080514.htm. Consultado el 1 de septiembre de 2008.

3. Ministerio de Salud. Garantías explicitas en salud. Guía clínica cáncer cervicouterino. 2005. Disponible en: http://www.minsal.cl/ici/guiasclinicas/CancerCervicouterino.pdf. Consultado el 25 de enero de 2007.

4. Organización Panamericana de la Salud. La OPS insta a que se adopte un nuevo método para la prevención del cáncer cérvicouterino. 2007. Disponible en: http://www.paho.org/Spanish/DD/PIN/ps070619.htm. Consultado el 1 de septiembre de 2008.

5. Organización Panamericana de la Salud. PAHO makes a call to the countries of the Americas for "that all the children count: the cervical cancer is preventive". 2007. Disponible en: http://www.paho.org/Spanish/DD/PIN/ps070308a.htm. Consultado el 1 de septiembre de 2008.

6. Kahn JA, Goodman E, Huang B, Slap GB, Emans SJ. Predictors of Papanicolaou smear return in a hospitalbased adolescent and young adult clinic. Obstet Gynecol 2003:101(3):490-9.

7. Ho V, Yamal JM, Atkinson EN, Basen-Engquist K, Tortolero Luna G, Follen M. Predictors of breast and cervical screening in Vietnamese women in Harris County, Houston, Texas. Cancer Nurs 2005;28(2):119-29; quiz 130-111.

8. McFarland DM. Cervical cancer and Pap smear screening in Botswana: Knowledge and perceptions. Int Nurs Rev 2003;50(3):167-75.

9. Lamadrid S. Conocimientos y temores de las mujeres chilenas con respecto a la prueba de Papanicolaou. Bol Of Sanit Panam 1996;121(6):542-9.

10. Lamadrid S. Aspectos socio-culturales de la sexualidad como factores obstaculizadores de la prevención secundaria del cáncer cervico-uterino. Cad Saúde Pública 1998:14(Supl 1):33-40.
11. Urrutia MT, Poupin L, Concha X, Vinales D, Iglesias C, Reyes V. ¿Por que las mujeres no se toman el Papanicolau?: Barreras percibidas por un grupo de mujeres ingresadas al programa de cáncer cervicouterino AUGE. Rev Chil Obstet Ginecol 2008:73(2):98-103.

12. Valenzuela MT, Miranda A. ¿Por que no me hago el Papanicolau? Barreras psicológicas de mujeres de sectores populares de Santiago de Chile. Rev Chil Salud Pública 2001:5(2-3):75-80.

13. Rosenstock I. Historical origins of the health belief model. In: Becker M (Ed.). The health belief model and personal health behavior. New Jersey: Slack CB. 1974. pp. 1-8.

14. Finfgeld DL, Wongvatunyu S, Conn VS, Grando VT, Riussell CL. Health belief model and reversal theory: A comparative analysis. J Adv Nurs 2003:43(3):288-97.

15. Institute of Medicine. Individuals and families: models and interventions. In: Institute of Medicine (Ed.). Health and behavior. The interplay of biological, behavioral and societal influences. Washington: National Academy of Press. 2001. pp. 183-240.

16. Rosenstock I, Derryberry M, Carriger B. Why people fail to seek poliomyelitis vaccination. Public Health Report 1959:74(2):98-103.

17. Urrutia MT. Development and testing of a questionnaire: beliefs about cervical cancer and Pap test in Chilean women. Tesis doctoral presentada a la Universidad de Miami (USA) como uno de los requisitos para obtener el grado de PhD. Diciembre, 2009.

18. Rosenstock I. The health belief model and preventive health behavior. In: M. Becker M (Ed.). The health belief model and personal health behavior. New Jersey: Slack CB.1974. (pp. 27-59).

19. Nunnaly J, Bernstein I. Factor analysis: The general model and variance. In: Nunnaly J, Bernstein I (Eds.). Psychometric theory: McGraw-Hill.1994. pp. 447-490.

20. Ministerio de Salud. Programa nacional de cáncer cervicouterino.2008. Disponible en: http://www.redsalud. gov.cl/temas_salud/proteccion/cancer2.html. Consultado el 17 de octubre de 2008.

21. McGovern PG, Lurie N, Margoli, KL, Slater JS. Accuracy of self-report of mammography and Pap smear in a low-income urban population. Am J Prev Med 1998;14(3):201-8.

22. Sawyer JA, Earp JA, Fletcher RH, Daye FF, Wynn TM. Accuracy of women's self-report of their last Pap smear. Am J Public Health 1989;79(8):1036-7.

23. Boyer LE, Williams M, Calliste LC, Marshall ES. Hispanic women's perceptions regarding cervical cancer screening. JOGNN 2001;30(2):240-5.

24. National Cancer Institute. Cancer risk: Understanding the puzzle. Cervical cancer-step1: Find out about cervical cancer risk. 2009. Disponible en: http://understandingrisk.cancer.gov/a_Cervical/01.cfm. Consultado el 18 de julio de 2009 .

25. Center for Disease Control and Prevention. Cervical cancer basic information. 2008. Disponible en: http:// www.cdc.gov/cancer/cervical/basic_info/. Consultado el 1 de septiembre de 2008.

26. National Cancer Institute. Understanding cervical cancer changes: A health guide for women. 2009. Disponible en: www.cancer.gov/cancertopics/understandin- 
gcervicalchanges/page2. Consultado el 18 de julio de 2009.

27. Brenna S, Hardy E, Zeferino L, Namura I. Knowledge, attitudes, and practices related to the Pap smear among women with cervical cancer. Cad Saúde Pública 2001;17(4):909-14.

28. Boonpongmane C, Jittanoon P. Predictors of Papanicolau testing in working women in Bangkok, Thailand. Cancer Nurs 2007;30(5):384-9.

29. Watkins MM, Gabali C, Winkleby M, Gaona E, Lebaron S. Barriers to cervical cancer screening in rural Mexico. Int J Ginecol Cancer 2002;12(5):475-9.

30. Tacken MA, Braspenning JC, Hermens RP, Spreeuwenberg PM, van den Hoogen $\mathrm{HJ}$, de Bakker $\mathrm{DH}$, et al. Uptake of cervical cancer screening in The Netherlands is mainly influenced by women's beliefs about the screening and by the inviting organization. Eur J Public Health 2007;17(2):178-85.

31. Taylor VM, Jackson JC, Tu SP, Yasui Y, Schwartz SM, Kuniyuki A, et al. Cervical cancer screening among Chinese Americans. Cancer Detect Prev 2002;26(2):139-45.
32. Holroyd E, Taylor Pilia, RE, Twinn SF. Investigating Hong Kong's Filipino domestic workers' healthcare behavior, knowledge, beliefs and attitudes towards cervical cancer and cervical screening. Women Health 2003;38(1):69-82.

33. Fernandez MA, Tortolero Luna G, Gold RS. Mammography and Pap test screening among low-income foreign-born Hispanic women in USA. Cad Saude Publica 1998;14(Supp 3):133-147.

34. Bingham A, Bishop A, Coffey P, Winkler J, Bradley J, Dzuba I, et al. Factor affecting utilization of cervical cancer prevention services in low resource setting. Salud Pública de México 2003;45(supl 3):408-16.

35. Antshel K. Integrating culture as a means of improving treatment adherence in the Latino population. Psychol Health Med 2002;7(4): 435-49.

36. Real Academia de la Lengua Española. Diccionario online. Disponible en: http://buscon.rae.es/drael/ SrvltConsulta?TIPO_BUS=3\&LEMA=creencia. Consultado el 29 de junio de 2011. 